\title{
Lidamycin decreases CD133 expression in hepatocellular carcinoma via the Notch signaling pathway
}

\author{
YI CHEN $^{1 *}$, WENWEI SUN $^{1 *}$, RAN HE $^{1}$, FEIYAN ZHANG ${ }^{1}$, HONGYU WANG $^{1}$, \\ PANHONG LI ${ }^{1}$, RONG-GUANG SHAO ${ }^{2}$ and XIAOYU XU ${ }^{1}$ \\ ${ }^{1}$ College of Pharmaceutical Sciences, Southwest University, Chongqing 400715; ${ }^{2}$ Laboratory of Oncology, \\ Ministry of Health Key Laboratory of Antibiotic Bioengineering, Institute of Medicinal Biotechnology, \\ Chinese Academy of Medical Sciences and Peking Union Medical College, Beijing 100050, P.R. China
}

Received August 6, 2016; Accepted September 13, 2017

DOI: $10.3892 / 01.2017 .7248$

\begin{abstract}
Cluster of differentiation (CD)133 is considered a molecular marker of cancer stem cells in hepatocellular carcinoma. In the present study, the effect of lidamycin (LDM) on CD133 expression in hepatocellular carcinoma (Huh7 cells) was evaluated and the potential molecular mechanism was investigated. Flow cytometry analysis, as well as sorting, sphere formation and western-blot assays, were performed in vitro to explore the effects of LDM on CD133 expression. A subcutaneous tumor model in nude mice was used to observe the effects of LDM on tumor volume and CD133 protein in vivo. To investigate the potential underlying molecular mechanism, Notch signaling pathway activity was detected by western blot analysis and reverse transcription-quantitative polymerase chain reaction. The proportion of $\mathrm{CD}_{133^{+}}$cells and the expression of $\mathrm{CD} 133$ protein were revealed to be downregulated by LDM. Sphere formation of sorted $\mathrm{CD} 133^{+}$cells was suppressed 7 days after LDM treatment. In addition, LDM inhibited tumor volume formed from sorted $\mathrm{CD}_{133}{ }^{+}$cells and CD133 protein level in vivo. LDM decreased the mRNA level of NOTCH1, Hes1 (Hes family BHLH transcription factor 1) and Heyl (Hes-related family BHLH transcription factor with YRPW motif 1) genes; consequently, the protein expression of NOTCH1, Notch intracellular domain, Hes1 and Heyl was decreased by LDM. Downregulation of the Notch signaling pathway by LDM was enhanced through combination with $\mathrm{N}$-[N-(3,5-difluorophenacetyl)-L-alanyl]-S-phenylglycine
\end{abstract}

Correspondence to: Professor Yi Chen or Professor Xiaoyu Xu, College of Pharmaceutical Sciences, Southwest University, 1 Tiansheng Road, Chongqing 400715, P.R. China

E-mail: rachelcy@swu.edu.cn

E-mail:xuxiaoyu@swu.edu.cn

*Contributed equally

Abbreviations: LDM, lidamycin; HCC, hepatocellular carcinoma; CSCs, cancer stem cells; NICD, Notch intracellular domain

Key words: cancer stem cells, hepatocellular carcinoma, lidamycin, cluster of differentiation 133, Notch signaling pathway t-butyl ester. In brief, these data suggest that LDM suppresses CD133 expression via the Notch signaling pathway, indicating the potential mechanism of LDM on CD133 and the benefits for further clinical application.

\section{Introduction}

Hepatocellular carcinoma (HCC) is the most common type of liver cancer associated with a poor clinical outcome (1). Cancer stem cells (CSCs) have been identified in a number of solid tumors, including HCC (2-4). CSCs may lead to recurrence, drug-resistance and tumor formation in HCC (5). Cluster of differentiation (CD)133 is a membrane-bound pentaspan glycoprotein that has been identified as a surface biomarker in a variety of cancer types, including breast, colon, prostate, pancreatic, lung and liver carcinoma $(2,3,6-11)$. Although its function remains unclear, directly targeting CD133 has been demonstrated to be a potentially effective strategy for eliminating CSCs $(11,12)$. Thus, drug screens that target CSC markers may improve the efficacy of therapeutic strategies for the treatment of HCC.

Lidamycin (LDM), an anticancer antibiotic, has been demonstrated to exhibit distinct antitumor effects in various types of cancer, including liver, breast, pancreatic, colon, lung, gastric and brain cancer, as well as in lymphoma and myeloma (13). Furthermore, LDM is toxic to multi-drug resistant HCC cells (14). In our previous study, LDM was revealed to inhibit the expression of epithelial cell adhesion module (EpCAM) and the population of CSCs through regulating the glycogen synthase kinase (GSK) $3 \beta / \beta$-catenin signaling pathway (15). Thus, LDM has been demonstrated to be a potentially effective strategy to target CSCs in HCC. However, the effect of LDM on CD133 remains unknown.

In the present study, it was hypothesized that LDM may suppress CD133 expression. To investigate this, the proportion of $\mathrm{CD}_{133^{+}}$cells, the expression of $\mathrm{CD} 133$ protein and the sphere formation of sorted $\mathrm{CD} 133^{+}$cells were evaluated in vitro following LDM treatment. Subsequently, the tumor volume of enriched $\mathrm{CD}_{133^{+}}$cells and $\mathrm{CD} 133$ expression was measured in vivo following LDM treatment. Additionally, the effect of LDM on the Notch signaling pathway was investigated. 


\section{Materials and methods}

Cell culture. Human hepatocellular carcinoma Huh7 cells (American Type Culture Collection, Manassas, VA, USA) were cultured in Dulbecco's modified Eagle's medium, supplemented with $10 \%$ fetal bovine serum and $1 \%$ penicillin-streptomycin (Gibco; Thermo Fisher Scientific, Inc., Waltham, MA, USA) at $37^{\circ} \mathrm{C}$ and $5 \% \mathrm{CO}_{2}$.

Chemicals. LDM was supplied by Professor Lian-fang Jin of the Institute of Medicinal Biotechnology, Chinese Academy of Medical Sciences and Peking Union Medical College (Beijing, China), with a purity of $>95.0 \%$. LDM was prepared as previously described (15). In brief, $1 \mu \mathrm{M}$ LDM stock solution in saline was stored at $-80^{\circ} \mathrm{C}$ for in vitro experiments, and diluted in saline at $5 \mu \mathrm{g} / \mathrm{ml}$ prior to intravenous injection. $\mathrm{N}$-[N-(3,5-difluorophenacetyl)-L-alanyl]-S-phenylglycine t-butyl ester (DAPT; Sigma-Aldrich; Merck KGaA, Darmstadt, Germany) was prepared as a $20 \mathrm{mM}$ stock solution in DMSO, and stored at $-20^{\circ} \mathrm{C}$.

Flow cytometry analysis and sorting. FcR blocking reagent (Miltenyi Biotec $\mathrm{GmbH}$, Bergisch Gladbach, Germany) was added to $5 \times 10^{5}$ cells suspended in PBS and incubated at $4{ }^{\circ} \mathrm{C}$ for $10 \mathrm{~min}$. Subsequently, cells were separately stained with phycoerythrin-conjugated anti-human CD133 or anti-human IgG isotype antibodies (R\&D Systems, Inc., Minneapolis, MN, USA) for 30-40 min in $4^{\circ} \mathrm{C}$. IgG isotype was used as negative control. Ice-cold PBS was used as washing reagent. Flow cytometry analysis was performed on Accuri ${ }^{\mathrm{TM}}$ C6 (BD Biosciences, San Jose, CA, USA) using CFlow software (FCS3.0; BD Biosciences). Flow cytometry sorting was conducted using a BD FACSAria ${ }^{\mathrm{TM}}$ I (BD Biosciences).

Sphere formation assay. The sphere formation medium for HCC was created as previously described $(2,15)$. Sorted $\mathrm{CD}_{133^{+}}$cells in sphere formation medium were cultured in ultra-low attachment 24-well plates (Corning Inc., Corning, NY, USA) at a density of 5,000 cells/well. Sphere formation medium with 0.03 or $0.3 \mathrm{nM}$ LDM was refreshed twice a week. After 7 days of culture, spheres were counted and images were captured at a magnification of x200 using a light microscope (Eclipse TE2000-U; Nikon Corporation, Tokyo, Japan). The size of the spheres was analyzed using ImageJ software (version 1.51K; National Institutes of Health, Bethesda, MD, USA).

$R N A$ isolation and reverse transcription-quantitative polymerase chain reaction ( $R T-q P C R)$. Total mRNA was extracted from the Huh7 cells using TRIzol ${ }^{\boxplus}$ reagent (Invitrogen; Thermo Fisher Scientific, Inc.) and purified using acid phenol-chloroform. A SuperScript ${ }^{\mathrm{TM}}$ RT-PCR kit (Invitrogen; Thermo Fisher Scientific, Inc.) was used according to the manufacturer's protocol. RT-qPCR was performed in triplicate using SYBR ${ }^{\circledR}$ Green reagents (Bio-Rad Laboratories, Inc., Hercules, CA, USA) and CFX96 qPCR system (Bio-Rad Laboratories, Inc.) under the following conditions: $10 \mathrm{~min}$ at $95^{\circ} \mathrm{C}$, followed by 45 cycles of $15 \mathrm{sec}$ at $95^{\circ} \mathrm{C}$ and $30 \mathrm{sec}$ at $60^{\circ} \mathrm{C}$. The $\mathrm{Cq}$ values, analyzed by CFX Manager (Bio-Rad Laboratories, Inc.), were used for calculation of relative expression levels with the $2^{-\Delta \Delta C q}$ method (16). GAPDH was used as the reference gene; all primer sequences are presented in Table I.

Western blot analysis. Whole cell lysates were prepared as described previously (17). Briefly, cells were washed with ice-cold PBS and lysed with radioimmunoprecipitation assay lysis buffer (cat. no. 89900; Thermo Fisher Scientific, Inc.) containing protease inhibitor (Sigma-Aldrich; Merck KGaA). Protein concentrations were determined using a Bradford assay. Proteins $(\sim 30 \mu \mathrm{g} / \mathrm{lane})$ were separated by SDS-PAGE $(10 \%$ gel). Proteins were transferred onto a polyvinylidene difluoride membrane (Merck KGaA) blocked with dried non-fat skimmed milk (5\% in Tris-buffered saline containing Tween-20) at room temperature for $2 \mathrm{~h}$. The membranes were subsequently probed with primary mouse anti-CD133 (1:200 dilution; cat. no. 130-090-422; Miltenyi Biotec $\mathrm{GmbH}$ ), rabbit anti-Notch intracellular domain (NICD; 1:500 dilution; cat. no. 4147; Cell Signaling Technology, Inc., Danvers, MA, USA), rabbit anti-NOTCH1 (1:1,000 dilution; cat. no. 3447; Cell Signaling Technology, Inc.), rabbit anti-Hes1 (1:1,000 dilution; cat. no. 11988; Cell Signaling Technology, Inc.) and mouse anti- $\beta$-actin (1:3,000 dilution; cat. no. A3854; Sigma-Aldrich; Merck KGaA) antibodies overnight at $4^{\circ} \mathrm{C}$. The membranes were then incubated with horseradish peroxidase (HRP)-linked anti-rabbit secondary antibody (1:3,000 dilution; cat. no. 7074; Cell Signaling Technology, Inc.) or HRP-linked anti-mouse secondary antibody (1:3,000 dilution; cat. no. 7076; Cell Signaling Technology, Inc.) at room temperature for $2 \mathrm{~h}$. Enhanced chemiluminescence (ECL) was performed using ECL Western Blotting Substrate (cat. no. 32106; Pierce; Thermo Fisher Scientific, Inc.) and the ChemiImager 5500 imaging system (ProteinSimple, San Jose, CA, USA), according to the manufacturer's protocol. The intensity of the bands was analyzed by densitometry using the ChemiImager AlphaEaseFC ${ }^{\mathrm{TM}}$ software version 4.0 (ProteinSimple).

Animal care and ethical statement. Nude mice $(\mathrm{n}=18$, 50:50 male/female, aged 5-6 weeks, weighing 18-20 g) were obtained from the Chinese Academy of Military Medical Sciences (Beijing, China). They were housed in a room with a 12-h light/12-h dark cycle, ad libitum access to food and water, an ambient temperature of $22^{\circ} \mathrm{C}$. This study was carried out in strict accordance with the recommendations in the Guide for the Care and Use of Laboratory animals of the Chinese Academy of Medical Science [permit number, SYXK (Jing) 2010-0013]. The experimental protocols were approved by the Ethics Committee of the Institute of Medicinal Biotechnology at the Chinese Academy of Medical Science. To minimize suffering, sodium pentobarbital anesthesia (100 mg/kg i.p.) was administered prior to surgery. Mice were sacrificed at 21 days after $\mathrm{CO}_{2}$ injection.

Tumorigenicity assay in nude mice. Following cell separation using a MACS kit (Miltenyi Biotec $\mathrm{GmbH}$ ), according to the manufacturer's protocol, enriched $\mathrm{CD} 133^{+} \mathrm{Huh} 7$ cells $\left(3 \times 10^{5}\right.$ cells/mouse) were subcutaneously injected into each nude mouse. The mice were randomly separated into three groups $(n=6)$ : The control group was intravenously injected with an equal volume of saline; the LDM groups were intravenously 
Table I. List of polymerase chain reaction primers.

\begin{tabular}{|c|c|c|c|}
\hline Gene & Species & Forward primer & Reverse primer \\
\hline Notch1 & Human & 5'-CACTGTGGGCGGGTCC-3' & 5'-GTTGTATTGGTTCGGCACCAT-3' \\
\hline Hes 1 & Human & 5'-GTCAAGCACCTCCGGAAC-3' & 5'-CGTTCATGCACTCGCTGA-3' \\
\hline Hey1 & Human & 5'-TCTGAGCTGAGAAGGCTGGT-3' & 5'-CGAAATCCCAAACTCCGATA-3' \\
\hline GAPDH & Human & 5'-TGAAGGTCGGTGTGAACGG-3' & 5'-CGTGAGTGGAGTCATACTGGAA-3' \\
\hline
\end{tabular}

Hes1, Hes family BHLH transcription factor 1; Hey1, Hes-related family BHLH transcription factor with YRPW motif 1.

injected with 0.025 or $0.05 \mathrm{mg} / \mathrm{kg} \mathrm{LDM}$. All treatments were applied in a single injection, $24 \mathrm{~h}$ after cell transplantation. Tumor volume was examined bi-weekly and the mice were sacrificed 21 days after cell injection. Tumors were measured with a Vernier caliper and the volume was calculated using the following equation: $\mathrm{V}=1 / 2\left[\left(\mathrm{width}^{2}\right) \mathrm{x}\right.$ length].

Statistical analysis. The flow cytometry data are presented as the arithmetic mean. Other data are presented as the arithmetic mean \pm standard deviation, with all experiments repeated in triplicate. The data for LDM regarding the NOTCH1, NICD, Hes1 and Hey1 genes and protein levels were compared with the two independent-samples t-test. One-way analysis of variance was used for comparisons of the data when there were $>2$ groups, using SPSS software (version 21.0; IBM Corp., Armonk, NY, USA). $\mathrm{P}<0.05$ was considered to indicate a statistically significant difference.

\section{Results}

LDM decreases CD133 expression in vitro. CD133 is considered to be a surface marker of CSCs in $\operatorname{HCC}(2,4)$. As aforementioned, the $\mathrm{IC}_{50}$ of LDM was $0.3 \pm 0.1 \mathrm{nM}$ in the Huh7 cells treated for $48 \mathrm{~h}$ (data not shown). Thus, 0.03 and $0.3 \mathrm{nM}$ LDM was used in vitro. Using a flow cytometry assay, it was revealed that $0.3 \mathrm{nM}$ LDM decreased the population of $\mathrm{CD}_{133^{+}}$cells $(25.2 \pm 0.3 \%)$ compared with the control group $(37.8 \pm 0.4 \%)(\mathrm{P}<0.05)$, while there was no marked difference between $0.03 \mathrm{nM}$ LDM $(36.0 \pm 0.3 \%)$ and the control (Fig. 1A). Following treatment with 0.03 or $0.3 \mathrm{nM}$ LDM for 6,12 or $24 \mathrm{~h}$, proteins were collected for western blot analysis. The relative densitometry measurements of bands in $0.03 \mathrm{nM} \mathrm{LDM}$ for $12 \mathrm{~h}$ and $0.3 \mathrm{nM}$ LDM for 12 and $24 \mathrm{~h}(0.7 \pm 0.1,0.5 \pm 0.1$ and $0.3 \pm 0.1$, respectively) were lower than that of the control group. The results demonstrated that LDM significantly decreased the expression of CD133 protein in a dose- and time-dependent manner $(\mathrm{P}<0.05)$. At $6 \mathrm{~h}$, there was no marked difference between the LDM-treated groups and the control group (Fig. 1B and C). The data suggested that LDM may inhibit the formation of a population of $\mathrm{CD}_{133^{+}}$cells and CD133 protein expression.

Sphere formation of $\mathrm{CD} 133^{+}$cells is inhibited by LDM. Under non-adherent conditions, sphere formation has been demonstrated to resemble the characteristics of CSCs (18). $\mathrm{CD}_{133^{+}}$cells were separated using fluorescence-activated cell sorting and sphere formation was detected. The size of spheres formed from sorted CD133+ ${ }^{+}$cells was significantly diminished following exposure to LDM for 7 days (Fig. 2A). Additionally, LDM decreased the number of spheres compared with the control after 7 days $(\mathrm{P}<0.01$; Fig. $2 \mathrm{~B})$. It was notable that the proportion of inhibition of $0.03 \mathrm{nM}$ LDM was $49 \%$, while $0.03 \mathrm{nM}$ was 10 -fold less than the $\mathrm{IC}_{50}$. The data suggested that the cell spheres are highly sensitive to LDM in conditional culture systems.

LDM suppresses CD133 in vivo. To investigate the effect of $\mathrm{LDM}$ on CD133 in vivo, enriched $\mathrm{CD}_{133}{ }^{+}$cells were injected into the subcutaneous tissues of nude mice. Tumor volumes were restricted at 21 days following 0.025 and $0.05 \mathrm{mg} / \mathrm{kg} \mathrm{LDM}$ treatment, compared with control group ( $\mathrm{P}<0.05$; Fig. 3A). In vivo western blot analysis demonstrated that 0.025 and $0.05 \mathrm{mg} / \mathrm{kg}$ LDM effectively inhibited CD133 protein expression compared with the saline control, consistent with the aforementioned in vitro data $(\mathrm{P}<0.05$; Fig. $3 \mathrm{~B})$. Notably, there was no marked difference between the two concentrations of LDM in vivo.

Notch signaling pathway downregulation by LDM. The Notch signaling pathway has been revealed to be upregulated in CSCs and has subsequently been demonstrated as an effective target to eliminate CSCs $(4,19-21)$. The Notch signaling pathway has also been suggested to be involved in CD133 signaling (22). To investigate the mechanism of LDM on CD133 in HCC, activation of the Notch signaling pathway was evaluated. LDM decreased the mRNA level of NOTCH1, Hes1 and Heyl genes $(\mathrm{P}<0.05$; Fig. 4A). Additionally, NOTCH1 protein levels decreased following LDM treatment, decreasing the expression of downstream proteins, including NICD, Hes1 and Heyl (Fig. 4B). DAPT is a $\gamma$-secretase inhibitor, and is commonly used to inhibit Notch signaling. Therefore, DAPT was used with or without LDM separately. NOTCH1 was downregulated only by LDM and not by DAPT, as compared with the control. However, DAPT-induced abatement of NICD was enhanced by LDM via downregulating NICD protein expression. Thus, Hes1 and Hey1 proteins were restricted only by DAPT, or by DAPT combined with LDM (Fig. 4C). These data suggest that the inhibitory effect of LDM on CD133 may result from inhibited activity of the Notch signaling pathway.

\section{Discussion}

LDM has been demonstrated to exhibit potential antitumor effects in HCC; a previous study revealed that LDM decreased 
A

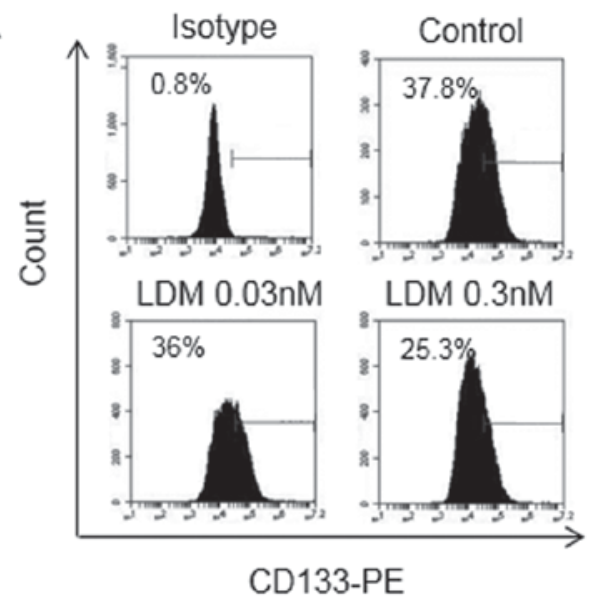

B

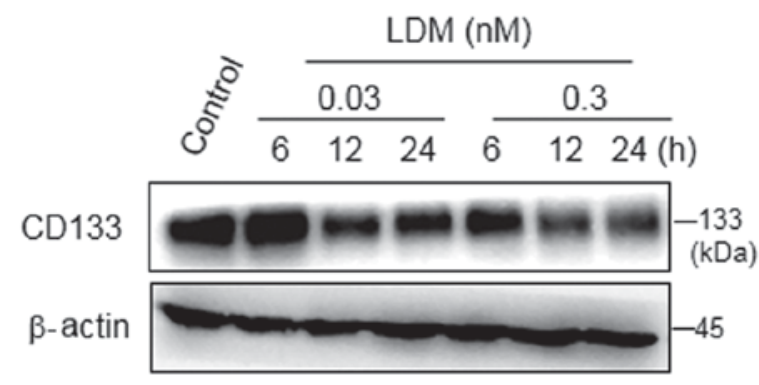

\section{C}

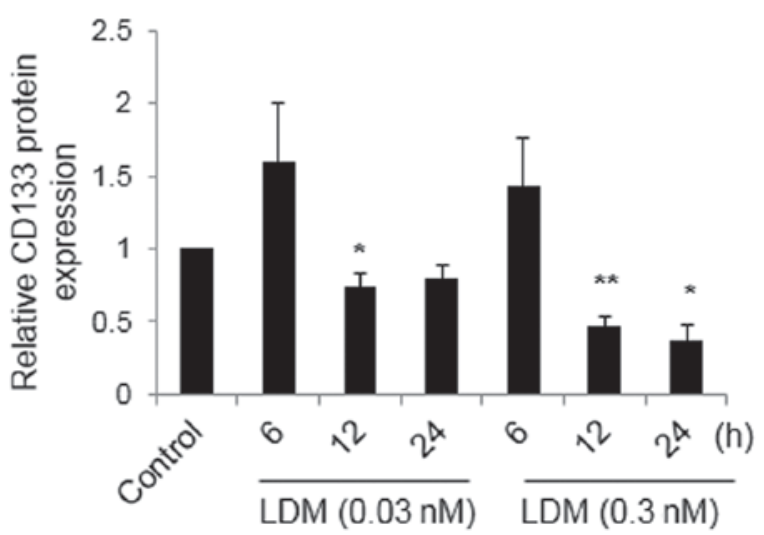

Figure 1. CD133 was decreased following LDM treatment in vitro. (A) The populations of CD133 cells were analyzed using flow cytometry following LDM treatment for $24 \mathrm{~h}$. The expression of CD133 protein was analyzed via (B) western blotting and (C) quantified for statistical analysis. *P<0.05 vs. control. ${ }^{* *} \mathrm{P}<0.01$ vs. control. Results are presented as the mean \pm standard deviation $(\mathrm{n}=3)$. LDM, lidamycin; CD, cluster of differentiation; PE, phycoerythrin.

A

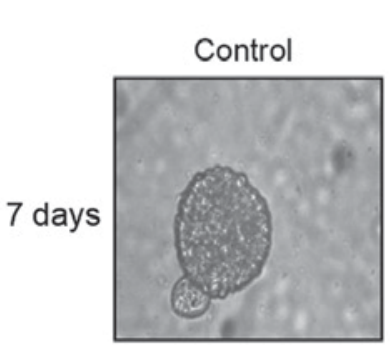

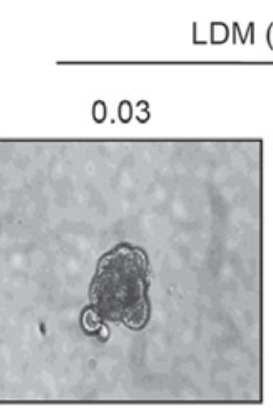

$\operatorname{LDM}(\mathrm{nM})$

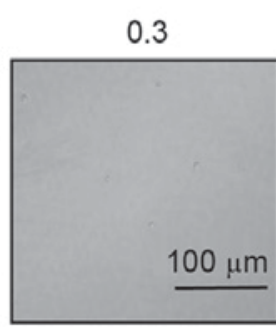

B

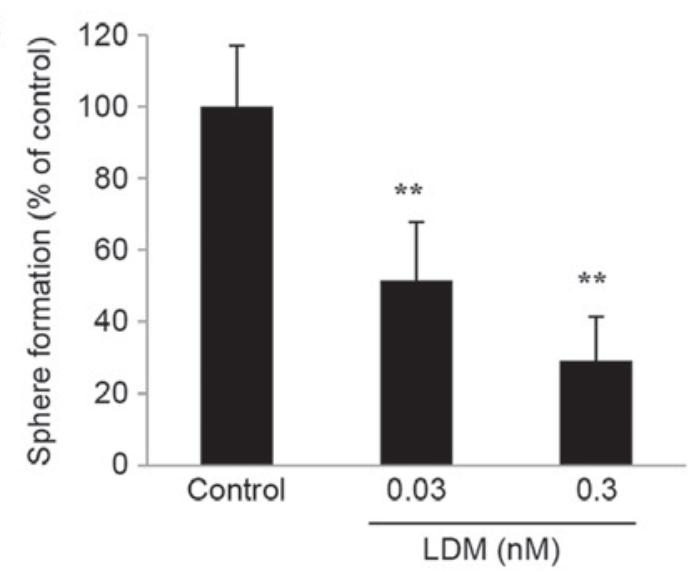

Figure 2. Sphere formation was inhibited by LDM. Spheres of sorted CD133+ cells were treated with LDM or saline (control) for 7 days. The (A) size and (B) number of spheres was detected after 7 days. ${ }^{* *} \mathrm{P}<0.01$ vs. control. Results are presented as the mean \pm standard deviation ( $\mathrm{n}=3$ ). Magnification, $\mathrm{x} 200$. LDM, lidamycin; $\mathrm{CD}$, cluster of differentiation.

the levels of embryonic stem cell-like genes (23). In addition, LDM inhibits EpCAM+ tumor initiating cells through the GSK3 $\beta / \beta$-catenin signaling pathway in HCC (15). The effects of LDM on CD133 in HCC were investigated. In the present study, LDM markedly inhibited CD133 expression of HCC in vitro and in vivo.
Although the function of CD133 is not entirely known, CD133 is considered an important marker of CSCs with numerous potential applications; direct downregulation of CD133 may be an effective strategy for the elimination of CSCs $(12,24)$. A previous study demonstrated that using antisense oligodeoxynucleotides to knock-down CD133 expression 
A

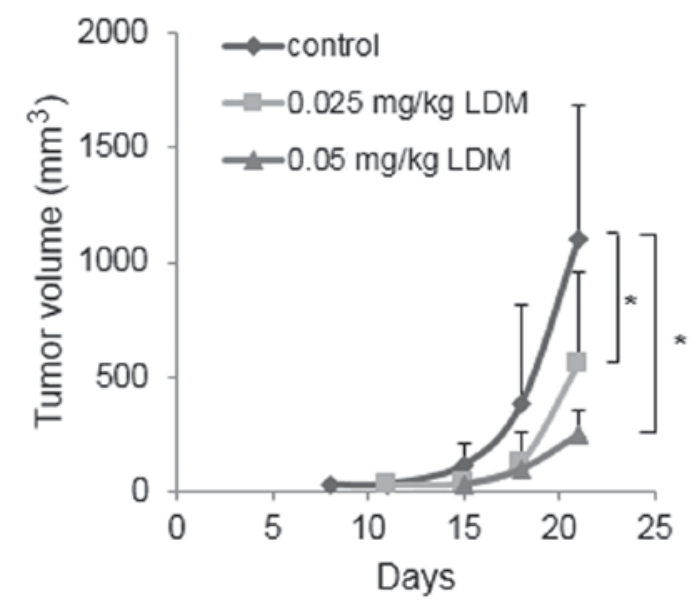

B

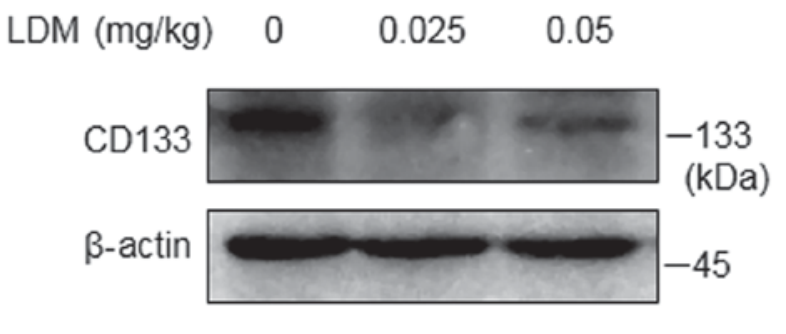

C

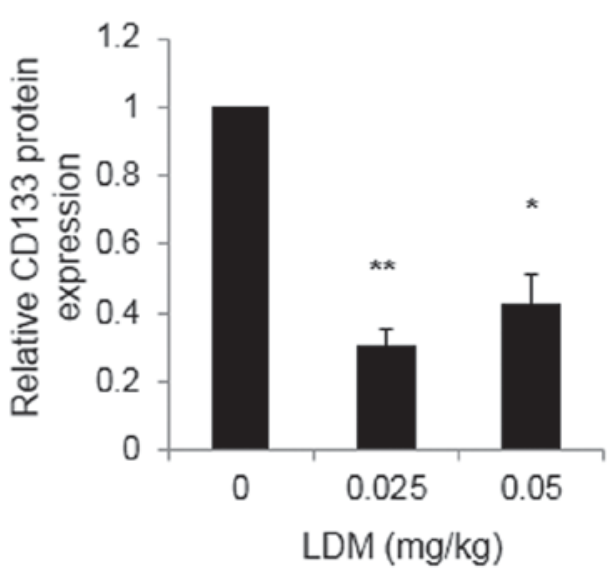

Figure 3. CD133 was inhibited by LDM in vivo. (A) The volumes of each tumor formed from sorted CD133 ${ }^{+}$cells were compared after 21 days. The (B) expression and (C) quantification of $\mathrm{CD} 133$ protein was analyzed via western blotting. ${ }^{*} \mathrm{P}<0.05$ vs. control. ${ }^{* *} \mathrm{P}<0.01$ vs. control. Results are presented as the mean \pm standard deviation $(n=6)$. LDM, lidamycin; CD, cluster of differentiation.

leads to a decrease in colony formation and to cell cycle arrest (24). Another group generated an anti-CD13/anti-CD133 bispecific antibody (BsAb) with cytokine-induced killer (CIK) cells to target CD133 CSCs, where by BsAb-CIK significantly inhibited tumor growth in HCC (12). In addition to direct targeting of CD133, the CD133-associated signaling pathway may be regulated, and it has been demonstrated that CD133 was decreased through the blockade of the Notch signaling pathway (25). The results of the present study suggested that LDM-mediated suppression of CD133 may proceed via the downregulation of Notch signaling, with data suggesting that, direct or indirect, the inhibition of CD133 may be an effective target for CSCs.

The Notch signaling pathway serves an important function in cell proliferation, self-renewal and differentiation. In canonical signaling, Notch ligands, including Jagged1, Jagged 2 or Delta-like 4, bind to Notch receptors (Notch1-4). Subsequently, intracellular cleavage is promoted in Notch receptors by $\gamma$-secretase, and NICD, which is the active form of Notch, is released and translocates to the nucleus where it binds to transcription factors. Thus, downstream genes are expressed, including Hes1 and Heyl (26). DAPT is a Notch signaling pathway inhibitor that decreases NICD levels via the inactivation of $\gamma$-secretase (27). Consistent with a previous study, the results of the present study demonstrated that LDM decreases the mRNA and protein levels of NOTCH1, compared with the control. Expression of NICD protein and the mRNA and protein levels of Hes1 and Hey1 were similarly decreased compared with the control. Additionally, LDM-induced decrease of NICD expression was enhanced in the presence of DAPT through the downregulation of NICD protein expression. Consequently, Hes1 and Hey1 protein were markedly decreased by DAPT combined with LDM. The results of the present study suggested that the inhibitory effect of LDM may suppress the activity of the canonical Notch signaling pathway.

In a previous study, LDM was revealed to downregulate embryonic stem cell-like genes: Octamer-binding transcription factor 4, SRY-box 2 and MYC proto-oncogene (23). Additionally, LDM has been demonstrated to decreases the expression of EpCAM, aldehyde dehydrogenase-positive cells, sphere formation in vitro, and inhibit tumor volume and incidence in vivo; this suggests that LDM may suppress Huh7 tumor initiating cells (15). The results of the present study demonstrated that LDM decreases the expression of CD133 in vitro and in vivo, supporting the function of LDM as an inhibitor of tumor growth and CSCs, and indicated the potential mechanism of LDM on CSCs as well as suggesting the benefits and clinical applications for HCC. CD133 and 
A

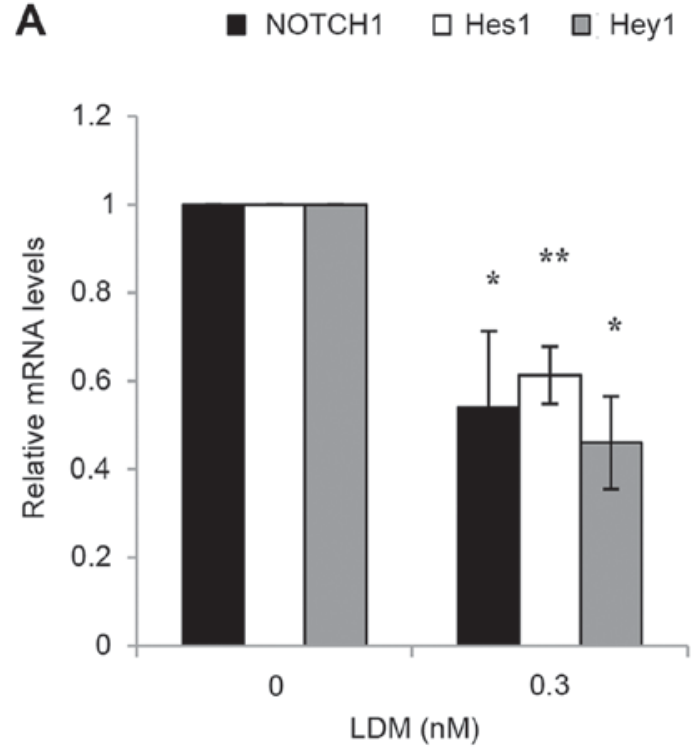

C

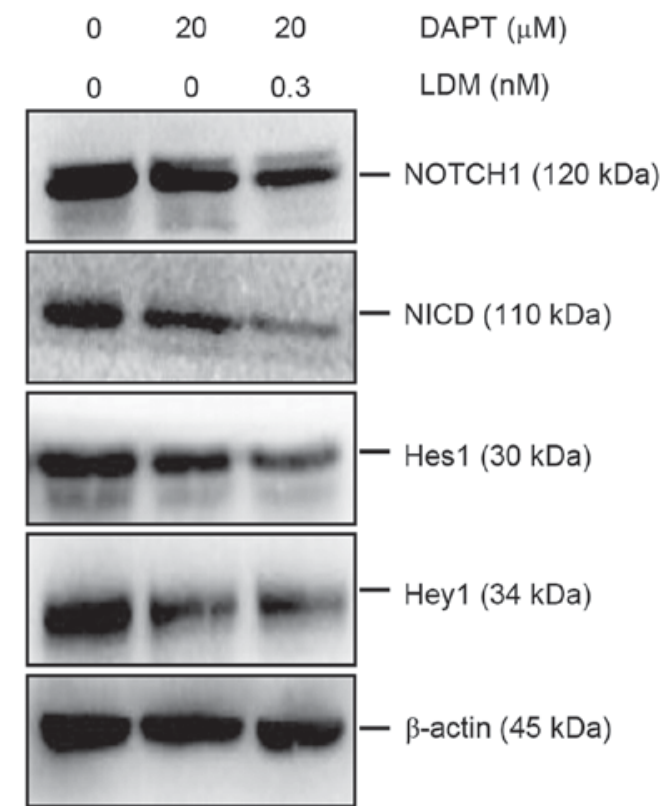

B $0 \quad 0.3 \quad \operatorname{LDM}(\mathrm{nM})$
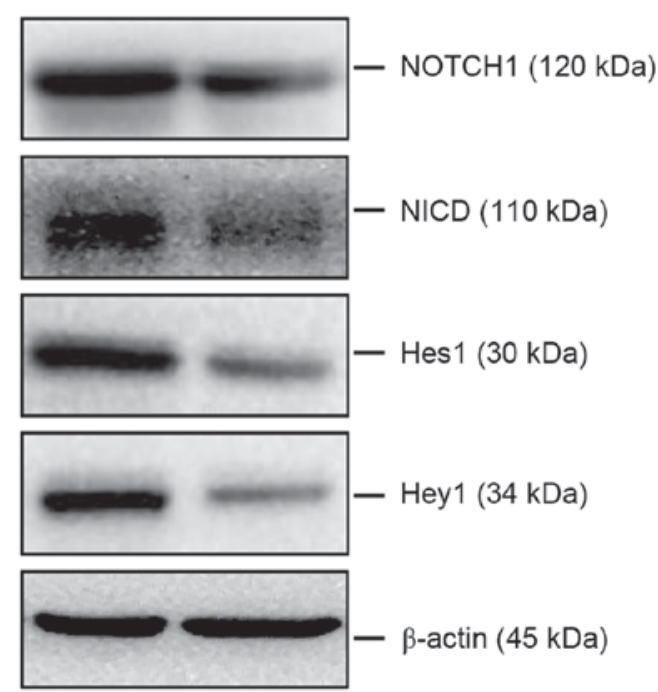

Figure 4. Regulation of LDM on the Notch signaling pathway. (A) The mRNA levels of NOTCH1, Hes1 and Hey1 were analyzed by reverse transcription-quantitative polymerase chain reaction following treatment with $0.3 \mathrm{nM}$ LDM for $12 \mathrm{~h}$. (B) Cells were treated with $0.3 \mathrm{nM} \mathrm{LDM}$ for $12 \mathrm{~h}$ and the expression of CD133 protein was analyzed via western blotting. (C) Cells were treated with $20 \mu \mathrm{M}$ DAPT in the absence or presence $0.3 \mathrm{nM}$ LDM for $12 \mathrm{~h}$. ${ }^{*} \mathrm{P}<0.05$ vs. control. ${ }^{* *} \mathrm{P}<0.01$ vs. control. Results are presented as the mean \pm standard deviation ( $\mathrm{n}=3$ ). LDM, lidamycin; $\mathrm{CD}$, cluster of differentiation; DAPT, $\mathrm{N}$-[N-(3,5-difluorophenacetyl)-L-alanyl]-S-phenylglycine t-butyl ester; NICD, Notch intracellular domain; Hes1, Hes family BHLH transcription factor 1; Hey1, Hes-related family BHLH transcription factor with YRPW motif 1.

EpCAM are the main CSC markers; however, there are many other markers, including CD90, CD13, $\mathrm{ABCG}_{2}$ and CD44, in which the effects exhibited by LDM require further investigation.

In conclusion, the results of the present study demonstrated that $\mathrm{LDM}$ decreases the $\mathrm{CD} 133^{+}$cell population, inhibits CD133 protein expression and suppresses the sphere formation of sorted $\mathrm{CD}_{133^{+}}$cells. In vivo experiments revealed that Huh7 tumor growth, formed from enriched CD133+ cells, was suppressed following LDM treatment. LDM also inhibits CD133 expression in tumor tissue. Furthermore, downregulation of the Notch signaling pathway may be a potential underlying molecular mechanism between LDM and CD133. These results support the requirement for further clinical evaluation of LDM.

\section{Acknowledgements}

The present study was supported by the National Natural Science Foundation of China (grant nos. 81402441 and 81773984), the China Postdoctoral Science Foundation (grant no. 2014M562272) and Fundamental Research Funds for the Central Universities (grant nos. XDJK2016C070 and XDJK2017D153). 


\section{References}

1. Dudeck O and Ricke J: Advances in regional chemotherapy of the liver. Expert Opin Drug Deliv 8: 1057-1069, 2011.

2. Chen Y, Yu D, Zhang H, He H, Zhang C, Zhao W and Shao RG: CD133(+)EpCAM(+) phenotype possesses more characteristics of tumor initiating cells in hepatocellular carcinoma Huh7 cells. Int J Biol Sci 8: 992-1004, 2012.

3. Ma S, Lee TK, Zheng BJ, Chan KW and Guan XY: CD133 ${ }^{+} \mathrm{HCC}$ cancer stem cells confer chemoresistance by preferential expression of the Akt/PKB survival pathway. Oncogene 27: 1749-1758, 2008.

4. Yamashita T, Ji J, Budhu A, Forgues M, Yang W, Wang HY, Jia H, Ye Q, Qin LX, Wauthier E, et al: EpCAM-positive hepatocellular carcinoma cells are tumor-initiating cells with stem/progenitor cell features. Gastroenterology 136: 1012-1024, 2009.

5. Chiba T, Iwama A and Yokosuka O: Cancer stem cells in hepatocellular carcinoma: Therapeutic implications based on stem cell biology. Hepatol Res 46: 50-57, 2016.

6. Wright MH, Calcagno AM, Salcido CD, Carlson MD, Ambudkar SV and Varticovski L: Brcal breast tumors contain distinct $\mathrm{CD}^{-} 4^{+} / \mathrm{CD} 24^{-}$and $\mathrm{CD} 133^{+}$cells with cancer stem cell characteristics. Breast Cancer Res 10: R10, 2008.

7. Soner BC, Aktug H, Acikgoz E, Duzagac F, Guven U, Ayla S, $\mathrm{Cal} \mathrm{C}$ and Oktem G: Induced growth inhibition, cell cycle arrest and apoptosis in $\mathrm{CD} 133^{+} / \mathrm{CD} 44^{+}$prostate cancer stem cells by flavopiridol. Int J Mol Med 34: 1249-1256, 2014.

8. Farhana L, Antaki F, Anees MR, Nangia-Makker P, Judd S Hadden T, Levi E, Murshed F, Yu Y, Van Buren E, et al: Role of cancer stem cells in racial disparity in colorectal cancer. Cancer Med 5: 1268-1278, 2016.

9. Weng CC, Kuo KK, Su HT, Hsiao PJ, Chen YW, Wu DC, Hung WC and Cheng KH: Pancreatic tumor progression associated with CD133 overexpression: involvement of increased TERT expression and epidermal growth factor receptor-dependent Akt activation. Pancreas 45: 443-457, 2016.

10. Koren A, Rijavec M,Kern I, Sodja E, Korosec P and Cufer T: BMI1, ALDH1A1, and CD133 transcripts connect epithelial-mesenchymal transition to cancer stem cells in lung carcinoma. Stem Cells Int 2016: 9714315, 2016

11. Ma S, Chan KW, Lee TK, Tang KH, Wo JY, Zheng BJ and Guan XY: Aldehyde dehydrogenase discriminates the CD133 liver cancer stem cell populations. Mol Cancer Res 6: 1146-1153, 2008.

12. Huang J, Li C, Wang Y, Lv H, Guo Y, Dai H, Wicha MS, Chang AE and Li Q: Cytokine-induced killer (CIK) cells bound with anti-CD3/anti-CD133 bispecific antibodies target CD133(high) cancer stem cells in vitro and in vivo. ClinImmunol 149: 156-168, 2013.

13. Shao RG and Zhen YS: Enediyne anticancer antibiotic lidamycin: Chemistry, biology and pharmacology. Anticancer Agents Med Chem 8: 123-131, 2008.
14. Shi YK, Wu SY, Huang YH and Zhen YS: Chemosensitivity of mdrl gene overexpressed multidrug resistant cancer cells to lidamycin. Yao Xue Xue Bao 41: 1146-1151, 2006 (In Chinese).

15. Chen Y, Yu D, Zhang C, Shang B, He H, Chen J, Zhang H, Zhao W, Wang Z, Xu X, et al: Lidamycin inhibits tumor initiating cells of hepatocellular carcinoma Huh7 through GSK3 $\beta / \beta$-catenin pathway. Mol Carcinog 54: 1-8, 2015.

16. Griffioen AW, Mans LA, de Graaf AMA, Nowak-Sliwinska P1, de Hoog CLMM, de Jong TAM, Vyth-Dreese FA, van Beijnum JR, Bex A and Jonasch E: Rapid angiogenesis onset after discontinuation of sunitinib treatment of renal cell carcinoma patients. Clin Cancer Res 18: 3961-3971, 2012.

17. Zhang H, Zhang S, He H, Zhao W, Ren K, Chen J and Shao RG RasGAP-derived peptide 38GAP potentiates the cytotoxicity of cisplatin through inhibitions of Akt, ERK and NF- $\mathrm{BB}$ in colon carcinoma HCT116 cells. Cancer Lett 308: 62-70, 2011.

18. Cao L, Zhou Y, Zhai B, Liao J, Xu W, Zhang R, Li J, Zhang Y, Chen L, Qian H, et al: Sphere-forming cell subpopulations with cancer stem cell properties in human hepatoma cell lines. BMC Gastroenterol 11: 71, 2011.

19. de Sousa EM, Vermeulen L, Richel D and Medema JP: Targeting Wnt signaling in colon cancer stem cells. Clin Cancer Res 17: 647-653, 2011.

20. Li Y, Zhang T, Korkaya H, Liu S, Lee HF, Newman B, Yu Y, Clouthier SG, Schwartz SJ, Wicha MS and Sun D: Sulforaphane, a dietary component of broccoli/broccoli sprouts, inhibits breast cancer stem cells. Clin Cancer Res 16: 2580-2590, 2010.

21. Xu W, Lin H, Zhang Y, Chen X, Hua B, Hou W, Qi X, Pei Y,Zhu X, Zhao Z and Yang L: Compound kushen injection suppresses human breast cancer stem-like cells by down-regulating the canonical Wnt/ $\beta$-catenin pathway. J Exp Clin Cancer Res 30: 103, 2011.

22. Wu D and Pan W: GSK3: A multifaceted kinase in Wnt signaling. Trends Biochem Sci 35: 161-168, 2010.

23. Zhen HY, He QH, Zhen YZ, Wang SL, Liu YN, Wu WH, Zhang XY, Lu AL and Shen L: Inhibition of mouse embryonic carcinoma cell growth by lidamycin through down-regulation of embryonic stem cell-like genes Oct4, Sox 2 and Myc. Invest New Drugs 29: 1188-1197, 2011.

24. Yao J,Zhang T,Ren J, Yu M and Wu G: Effect of CD133/prominin-1 antisense oligodeoxynucleotide on in vitro grow th characteristics of Huh-7 human hepatocarcinoma cells and U251 human glioma cells. Oncol Rep 22: 781-787, 2009.

25. Fan X, Khaki L, Zhu TS, Soules ME, Talsma CE, Gul N, Koh C, Zhang J, Li YM, Maciaczyk J, et al: NOTCH pathway blockade depletes CD133-positive glioblastoma cells and inhibits growth of tumor neurospheres and xenografts. Stem Cells 28: 5-16, 2010.

26. Crabtree JS, Singleton CS and Miele L: Notch signaling in neuroendocrine tumors. Front Oncol 6: 94, 2016.

27. Wang J, Ye Z, Zheng S, Chen L, Wan Y, Deng Y and Yang R: Lingo-1 shRNA and Notch signaling inhibitor DAPT promote differentiation of neural stem/progenitor cells into neurons. Brain Res 1634: 34-44, 2016. 